\title{
63. Internal Friction of Cold-worked Gold and Copper at Low Temperatures
}

\author{
By Ryukiti R. HASIGUTi and Sigeo OKUdA \\ Department of Metallurgy, University of Tokyo \\ (Comm. by T. Mishima, M.J.A., June 12, 1959)
}

The internal friction of metals is one of the powerful methods to study the lattice imperfections of metal crystals. On the other hand, there are many unsolved problems at present concerning the ways in which the lattice imperfections are produced and the ways in which they affect the properties of metals.

The purpose of the present research is to obtain quantitative knowledge on these problems by means of low frequency internal frictions. Since most of the lattice defects, which we are interested in, disappear at or below room temperature, it is necessary for us to perform the measurements at temperatures low enough to prevent the disappearance of the defects. We constructed, therefore, an apparatus to measure the internal friction and dynamic modulus in vacuum at low temperatures $\left(-180 \sim 100^{\circ} \mathrm{C}\right)$, using an inverted torsion pendulum method.

The specimens used were polycrystalline wires of $\mathrm{Au}(99.999 \%$ pure) and $\mathrm{Cu}(99.99 \%$ pure), 0.3 to $0.8 \mathrm{~mm}$ in diameter and about $10 \mathrm{~cm}$ in length. The specimen was first annealed, and fixed in the torsion pendulum. Then it was deformed in situ by torsion or tension at room temperature or at liquid nitrogen temperature. The internal frictions were measured as a function of temperatures from -180 to $80^{\circ} \mathrm{C}$. The effects of quenching were also investigated to compare with those of plastic deformation.

The experimental results. Specimens deformed at room temperature. A specimen of $\mathrm{Au}$ was deformed by torsion at room temperature, and then the internal friction was measured as a function of temperatures. About 20 minutes at room temperature were needed, before the specimen was refrigerated after plastic deformation. The results of measurements are shown in Fig. 1 . The peaks $P_{1}$ and $\mathrm{P}_{3}$ are seen at about $-140^{\circ} \mathrm{C}$ and about $-60^{\circ} \mathrm{C}$ respectively for the vibrational frequency of $1 \mathrm{cps}$. These peaks disappeared after annealing at room temperature (Fig. 1). It is expected from the shape of the curve at low temperatures that there is one more peak $\mathrm{P}_{0}$ at a temperature lower than the temperature range of our measurements. The heights of $P_{1}$ and $P_{3}$ increase at first with increasing amount of cold-work and then they decrease after passing a maximum 
or level off as the degree of cold-work is increased.

There are some differences between the annealing behaviours of $P_{1}$ and $\mathrm{P}_{3} . \quad \mathrm{P}_{3}$ decays at room temperature and disappears after about $10 \mathrm{hrs}$. On the other hand, on annealing at room temperature, the height of $\mathrm{P}_{1}$ increases at first and then

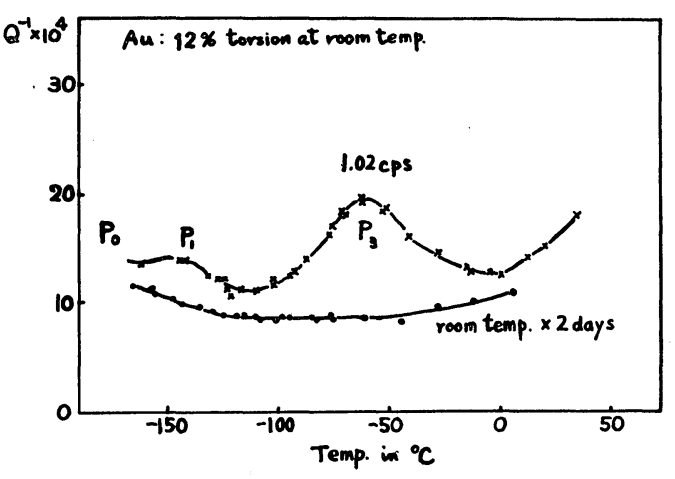

Fig. 1 decreases through a maximum. The time required for the disappearance of $\mathrm{P}_{1}$ is much longer than the time required for that of $\mathrm{P}_{3}$.

Specimens deformed at liquid nitrogen temperature. A specimen of Au was deformed at liquid nitrogen temperature and the internal friction was measured with increasing temperature, starting from the liquid nitrogen temperature. The results are shown by the solid curve

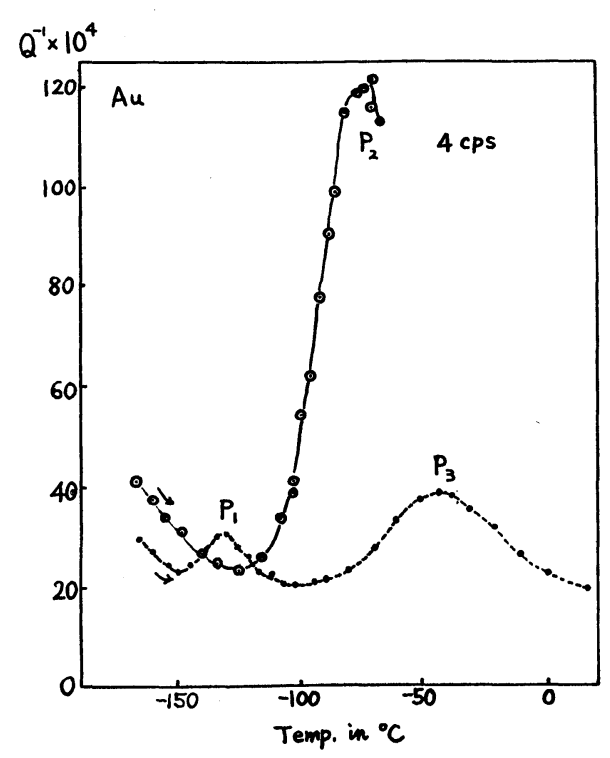

Fig. 2 in Fig. 2. Now a very large peak $\mathrm{P}_{2}$ appeared at a temperature a little lower than $\mathrm{P}_{3}$. This peak grew and decayed at about the peak temperatures. The height of this peak showed a maximum like $\mathrm{P}_{1}$, when it was measured as a function of the degrees of plastic deformation.

When the specimen is warmed up to about $-20^{\circ} \mathrm{C}$ after low temperature deformation, the internal friction versus temperature curve resumes the similar shape as that of the specimen deformed at room temperature (see dashed curve in Fig. 2).

We can obtain the knowledge on the change in dynamic modulus from measurements of the frequencies of free vibration. The change in these frequencies was measured along with the change in internal friction. The results are shown in Fig. 3. It is noticed from the figure that the growth and the decay of $\mathrm{P}_{2}$ internal friction peak correspond roughly to the increase and the decrease, respectively, of the dynamic modulus.

The specimen deformed at liquid nitrogen temperature was warmed 
up to a temperature around the foot of $\mathrm{P}_{2}$, and holding the specimen at the temperature, the internal friction and the dynamic modulus were measured against the holding time. Thus the curves shown in Fig. 4 were obtained. These curves show clearly what is mentioned above concerning the correspondence between the behaviours of internal friction and dynamic modulus. There is a shoulder shown by an arrow on the internal friction versus time curve,

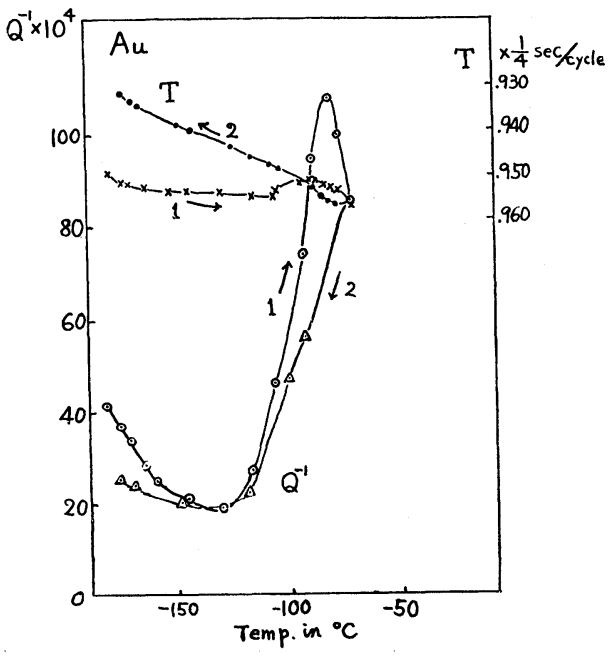

Fig. 3

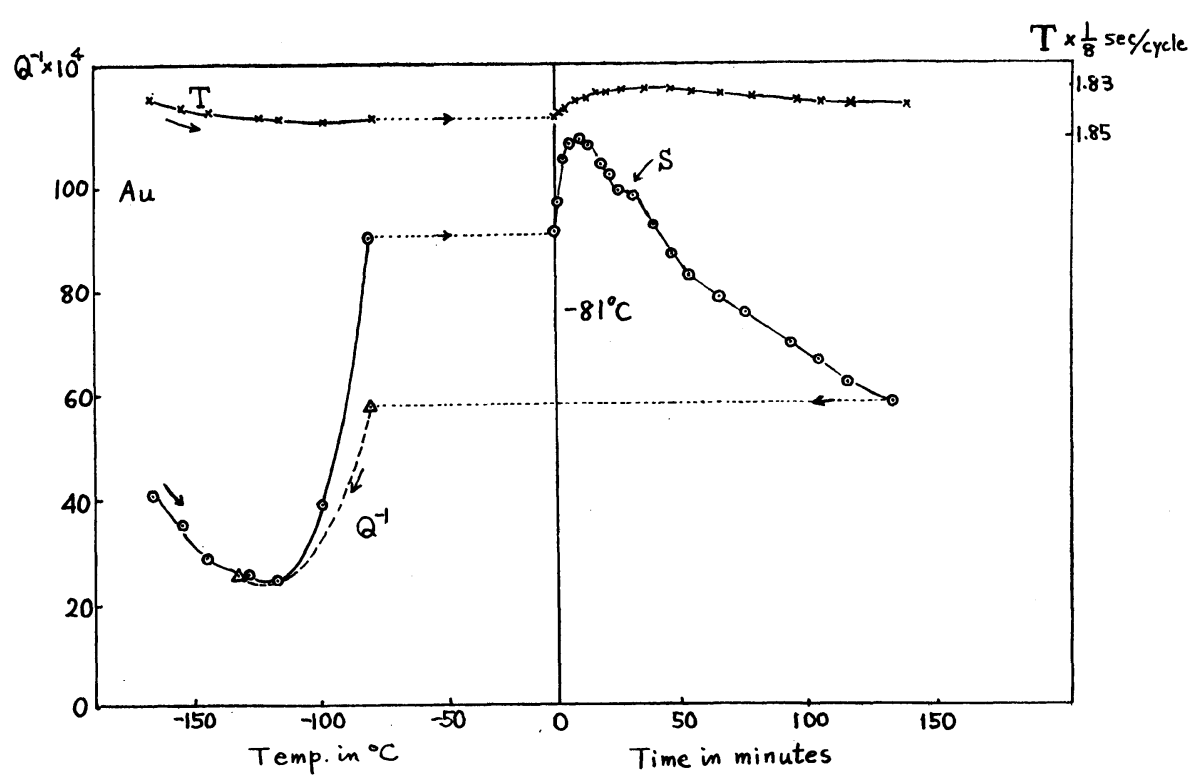

Fig. 4

and it is considered that this shoulder may be caused by the beginning of growth of $\mathrm{P}_{3}$.

The behaviours of $\mathrm{Cu}$ specimens are essentially the same as those of $\mathrm{Au}$ specimens.

Summary of the results. What have been shown above are only a part of the results obtained in our laboratory. Considering our whole results so far obtained, the behaviours of these peaks are summarized as follows. 
(i ) As far as the behaviours of internal friction are concerned, the state of the specimen deformed at liquid nitrogen temperature and annealed at room temperature is the same as that of the specimen deformed at room temperature.

( ii ) Three peaks $\mathrm{P}_{1}, \mathrm{P}_{2}$, and $\mathrm{P}_{3}$ appear after plastic deformation. These peaks are not peculiar to the $\mathrm{Au}(99.999 \%)$ specimens, but they appear also in the $\mathrm{Cu}(99.99 \%)$ specimens.

(iii) These three peaks do not appear immediately after deformation, but appear in certain respective annealing stages. These grow at first and then decay as the annealing proceeds.

(iv) The growth and decay of $\mathrm{P}_{2}$ and the growth of $\mathrm{P}_{1}$ are accompanied by remarkable changes in dynamic modulus. The modulus increases and decreases through a maximum, as the internal friction peak grows and decays, although the maximum of the modulus is reached a little later than the maximum of the internal friction.

( v ) The maximum heights of these peaks increase with increasing deformation at first, and then decrease or saturate at larger deformation. The peak temperatures are independent of the degrees of deformation, but dependent of the frequencies of measurements.

(vi) Activation energies are obtained from the frequency dependence of peak temperatures which will be referred to as the peak shift in the following table. The introduction of vacancies by quenching does not give rise to these peaks.

(vii) Activation energies and other important data are shown in the following table.

\begin{tabular}{|c|c|c|c|}
\hline $\mathrm{Au}$ & $P_{1}$ & $\mathrm{P}_{2}$ & $\mathrm{P}_{3}$ \\
\hline $\begin{array}{l}\text { Activation energy obtained from } \\
\text { the peak shift } E(\mathrm{eV})\end{array}$ & 0.22 & 0.34 & 0.37 \\
\hline Relaxation time $\quad \tau_{0}(\mathrm{sec})$ & $8 \times 10^{-10}$ & $5 \times 10^{-10}$ & $3 \times 10^{-10}$ \\
\hline Peak shape & $\begin{array}{l}\text { nearly single } \\
\text { relaxation }\end{array}$ & $\begin{array}{l}\text { nearly single } \\
\text { relaxation (?) }\end{array}$ & $\begin{array}{l}\text { not single } \\
\text { relaxation }\end{array}$ \\
\hline $\begin{array}{c}\text { Activation energy of growth } \\
\text { process of the peak }\end{array}$ & - & $\sim 0.45$ & - \\
\hline $\begin{array}{l}\text { Activation energy of decay } \\
\text { process of the peak }\end{array}$ & $\sim 0.7$ & $\sim 0.5$ & $\sim 1$ \\
\hline $\begin{array}{l}\text { Temperature at which the peak } \\
\text { growth is accelelated }\end{array}$ & $\sim-10^{\circ} \mathrm{C}$ & $\sim-150^{\circ} \mathrm{C}$ & $\sim-80^{\circ} \mathrm{C}$ \\
\hline $\mathrm{Cu}$ & $\mathrm{P}_{1}$ & $\mathrm{P}_{2}$ & $\mathrm{P}_{3}$ \\
\hline $\begin{array}{l}\text { Activation energy obtained from } \\
\text { the peak shift } \quad E(\mathrm{eV})\end{array}$ & 0.25 & 0.3 & 0.46 \\
\hline Relaxation time $\quad \tau_{0}(\mathrm{sec})$ & $3 \times 10^{-10}$ & $2 \times 10^{-10}$ & $5 \times 10^{-11}$ \\
\hline
\end{tabular}

Discussion. The theories of the internal friction hitherto proposed can not explain these results. We propose, therefore, tentatively the 
following theory. This theory can explain all our results consistently, and it does not contradict any other experimental results so far obtained.

It is proposed that the dislocations produced by plastic deformation are pinned down in the process of annealing by the point defects produced during the plastic deformation. A dislocation segment which contains a pinning point has a certain probability to be torn off the pinning point by the help of thermal energy. When the frequency of the tearing off process coincides with the external vibrational frequency of the specimen, there appears an internal friction peak as a relaxation process. It is considered that single vacancies, single interstitial atoms, and divacancies may pin down dislocations, and they give rise to the peaks $P_{1}, P_{2}$, and $P_{3}$ respectively.

According to this model, the activation energies obtained from the peak shifts correspond to the maximum interaction energies between a dislocation and respective point defects, and the activation energies of the growth processes of peaks are the activation energies for the migrations of respective point defects. The activation energies of decay processes of peaks are somewhat complicated.

The detail of this research will be published later elsewhere. 